\section{Revisión de enfoques académicos en salud global: un marco analítico}

\author{
Academic review of global health approaches: \\ an analytical framework
}

\section{Revisão de enfoques acadêmicos em saúde global: um marco analítico}

${ }^{1}$ Facultad Nacional de Salud Pública, Universidad de Antioquia, Medellín, Colombia.

Correspondencia A. Franco-Giraldo Facultad Nacional de Salud Pública, Universidad de Antioquia.

Calle 62, n. 52-59, Oficina 303, Medellín, Colombia. alvarofrancogiraldo@ hotmail.com

\begin{abstract}
In order to identify perspectives on global health, this essay analyzes different trends from academia that have enriched global health and international health. A database was constructed with information from the world's leading global health centers. The search covered authors on global diplomacy and global health and was performed in PubMed, LILACS, and Google Scholar with the key words "global health" and "international health". Research and training centers in different countries have taken various academic approaches to global health; various interests and ideological orientations have emerged in relation to the global health concept. Based on the mosaic of global health centers and their positions, the review concludes that the new concept reflects the construction of a paradigm of renewal in international health and global health, the pre-paradigmatic stage of which has still not reached a final version.
\end{abstract}

Global Health; Social Justice; Public Policies
Alvaro Franco-Giraldo ${ }^{1}$

\section{Resumen}

Con el fin de establecer perspectivas de la salud global, este ensayo analiza diferentes tendencias que han enriquecido, desde lo académico, la salud global y la salud internacional. Se construyó una base de datos con información sobre los principales y más importantes centros de salud global en el mundo. Para la búsqueda se tuvieron en cuenta autores de diplomacia global y salud global. La búsqueda se hace en PubMed, en LILACS y en Google Scholar con las palabras clave: "salud global" y "global health". Varios enfoques académicos se han perfilado acerca de la salud global por parte de centros de formación e investigación, según diferentes países. A su vez, emergen varios intereses y orientaciones ideológicas, detrás del concepto de salud global. Se concluye del variopinto mosaico de centros de salud global y sus posturas, que el nuevo concepto va tras la construcción de un paradigma de renovación en la salud internacional y en la salud mundial, que en su estadio pre-paradigmático no encuentra todavía una versión final.

Salud Global; Justicia Social; Políticas Públicas 


\section{Introducción}

El argot académico se ha visto inspirado por diversos centros e institutos de salud global, principalmente en Europa y en América. Los grupos anglosajones, pioneros en este campo, han desarrollado varias iniciativas en salud global, como se podrá apreciar en este artículo. El propósito de este ensayo es explorar las tendencias en salud global y los centros en el área, con sus diferentes perspectivas ideológicas y políticas, y no sólo académicas.

Los enfoques de salud global se van perfilando desde las concepciones de las ciencias sociales, pero en muchos casos, como veremos, algunas versiones de la salud global corren el riesgo de quedarse en una visión "demasiado sanitaria" 1, muy parecida a la salud internacional de viejo cuño 2,3 y, en otros casos, se dejan de lado los conceptos de transdisciplinariedad, tran-sectorialidad y transnacionalización, así como la presencia de nuevos actores 4 que la globalización ha impuesto. La salud global se construye poco a poco, como una disciplina académica que encara los problemas de la salud mundial y sus determinantes, mediante acciones globales o trasnacionales en pro de la salud y de la equidad global, pero con miradas disímiles desde las universidades, centros de investigación y centros de salud global.

Varias entidades académicas en el mundo vienen hablando de salud global y cada una la entiende a su manera, de acuerdo con su visión del mundo y la concepción de la salud pública, dada por el contexto en que se está inmerso. En general, la salud global se caracteriza como un proceso de salud poblacional dinámico, el cual depende de unos determinantes amplios que rebasan las propias fronteras de los países. Se pueden mencionar algunos de ellos 5: los modelos de desarrollo, el comercio, el medioambiente, la innovación tecnológica, las telecomunicaciones, etc.

En particular, este artículo defiende una representación de salud global emancipadora, transformadora e ideológicamente incluyente, basada en postulados sociales 6,7. En tal sentido, auspicia el debate acerca de las diversas interpretaciones y razones que llevaron al surgimiento de este nuevo paradigma. Es este el propósito fundamental de esta disertación.

Finalmente, se debate alrededor de algunas interpretaciones de la salud global y la salud internacional y su relación con la globalización que las condiciona. Además, se hace énfasis en las razones que llevaron al surgimiento de este nuevo paradigma de la salud global y sus diversas corrientes académicas. Tres aristas se propusieron para esta revisión analítica de la salud global: los avances teóricos en relación con "justicia, equidad y salud global"; el debate sobre "la acción trasnacional y las políticas trans-sectoriales por los derechos"; "la visión holística y la fuerza de una conciencia global" de orden macro-social y estructural del mundo, que abre nuevas posibilidades y nuevos desafíos de tipo cognitivo, investigativo y político en la salud mundial.

\section{Antecedentes}

Ya es bien conocido el debate salud global- salud internacional 8, que para unos representa un área de innovación conceptual y práctica de la salud pública en el orbe, para otros es una discusión impertinente. No obstante, algunos otros prefieren asimilar los términos y pasar de largo en la discusión. Es el caso del texto emanado de las entrañas de la propia Organización Panamericana de la Salud (OPS), el trabajo sobre competencias regionales de salud pública, que define salud global/salud internacional: "la salud internacional/ global es el enfoque transdisciplinario que aborda la salud desde la perspectiva del derecho universal a la salud y el bienestar social. En un mundo globalizado e interconectado se requieren abordajes globales con implicaciones locales que consideren los determinantes sociales de la salud y que con equidad, ética y respeto por los derechos humanos, se avance en soluciones transnacionales, regionales y globales que mejoren la salud de todos los ciudadanos del mundo. Utiliza como herramientas las relaciones entre las autoridades sanitarias nacionales, a través de la diplomacia en salud y la cooperación técnica" 9 (p. 19).

Se podría hacer el ejercicio de confrontar definiciones de salud global ya conocidas, pero no es suficiente, porque no siempre expresan los enfoques y tendencias ideológicas que se esconden tras las mismas. Por otro lado, la disimilitud y variedad no permite llegar a conclusiones suficientes sobre el panorama de la salud global en el ámbito académico global y sus múltiples dimensiones.

No obstante, Velji \& Bryant 10 cotejan definiciones principales de algunas declaraciones de importantes grupos y autores de renombre. Así, citando a Frenk 11, de la Universidad de Harvard (Estados Unidos), reconoce algunas ideas e ideales sobre la salud global: "Hoy más que nunca, la salud mundial está en la necesidad de una ética renovada, la ética de la derechos universales, por lo que todo ser humano puede tener la oportunidad de lograr su potencial completo". Valores que según el autor han servido desde siempre a la salud pública, como campo de investigación y como un espacio para la acción. 
El Comité de Expertos para la Salud Global en los Estados Unidos 12 (p. 39) entiende la salud global como: "el objetivo de mejorar la salud de todas las personas en todas las naciones por promover el bienestar y la eliminación de enfermedades evitables, discapacidades y muertes" entre sus estrategias, más allá de las clásicas de la salud pública, intenta la comprensión de los determinantes de la salud, la investigación básica y aplicada sobre los factores de riesgo, etc.

Brown et al. 13 citando a Alma-Mata (p. 456), entienden la salud global como un campo de la práctica, la investigación y la educación, "centrado en las fuerzas sociales, económicas, políticas y culturales que le dan forma a través del mundo"; disciplina relacionada con las cuestiones inherentes a la salud que trascienden las fronteras nacionales y los diferentes efectos de la globalización.

De Nigel Crisp (2010, apud Vejil \& Brant 10) considera que "el término salud global abraza a todos aquellos problemas de salud que nos afectan a todos, ricos y pobres". En el discurso, es indudable que varía con respecto a versiones anteriores de la salud poblacional y de la salud internacional, parece haber un consenso en que la salud global enfatiza los conceptos de equidad, la justicia, la equidad, los derechos, la solidaridad, la compasión, el respeto mutuo; "es una nueva visión y una acción paradigmática que se basa en las ideas humanas, ideales y valores de la prestación de alta calidad de la salud para todos a nivel mundial (...), trasciende fronteras, etnias, castas y religiones. Y consagra la noción del bien global" 10 (p. 307).

En el medio académico internacional se ha definido por el Consortium of Universities for Global Health Executive Board la salud global como: un área de estudio, investigación y práctica que toma un lugar prioritario en la mejora de la salud y el logro de la equidad sanitaria a lo ancho de todo el mundo, con impacto en todas las poblaciones 14 . Esta definición, retomada de Jeffrey Koplan et al. 14, concluye que la salud mundial pone de relieve los problemas transnacionales de salud, sus determinantes y soluciones.

Koplan et al. 14 posicionan la salud global como parte de la salud pública, como una extensión del dominio disciplinario, en cuanto tiene que ver con acciones geográficas, ya bien definidas de la salud pública, y ven diferencias en la salud pública que ha tenido un enfoque más preventivo de su actividad en las poblaciones, mientras que a la salud global es mucho más comprensiva y le conciernen todas las estrategias encaminadas a mejorar la salud. Sin embargo, Fried et al. 15 disienten respecto a que haya una clara diferencia entre ambas disciplinas.
En sentido parecido se han expresado muchos otros autores 16.

En otra perspectiva, la salud pública global es un bien general, basado en un nuevo valor público propio de la época reciente, sustentado en una buen gobernanza 17, que toma en cuenta los derechos humanos y una filosofía altruista, política y ética. Para algunos más, se define 18 como un nuevo enfoque en un nuevo contexto, como una nueva conciencia sobre la salud, en el marco de la globalización. A ello habría que agregar que no puede ser puesto al servicio de los intereses globalizadores de la economía. Otros lo han referido como un área de la salud mental 19.

Otras visiones se han venido desarrollado sobre el tema. Jacobsen 20 empieza por relacionar salud global con el estudio de las contribuciones biológicas, sociales y ambientales a la salud y la enfermedad de las poblaciones, alrededor del mundo. Asimismo, diferencia salud global del concepto tradicional de salud internacional, referido al estudio de la salud de la población que vive en los países en desarrollo. Salud global es más bien entendido como la "salud transnacional".

Velji \& Bryant 21 nos hablan de una nueva disciplina “ética en salud global”, como la teoría y la práctica de la ética de una manera holística, informada por múltiples disciplinas que incluyen salud pública y poblacional, sistemas de salud, biotecnología y otras áreas de investigación científica; de ella forman parte otras disciplinas como la filosofía, la ética, la antropología, el derecho, etc. También Burke 22 defiende que "salud global" debe entenderse desde la óptica de lo moral.

En todos los casos se enfatiza en que la salud global entiende las determinaciones sociales y ambientales sobre la salud- enfermedad, más allá de la causa biológica. Asimismo, tiene el reto de trascender áreas de la salud internacional clásica (enfermedades infecciosas, nutrición, salud infantil, salud reproductiva, agua y saneamiento), para pasar a otras que requiere el mundo actual (envejecimiento, salud mental, soberanía alimentaria). También la salud global subraya el impacto de la pobreza, la cultura y la globalización económica sobre la salud. El enfoque global de la salud internacional se torna muy importante para abordar temas tan complejos como el calentamiento de la tierra, el agotamiento de la capa de ozono, la disposición de desechos tóxicos y radioactivos, el deterioro de la calidad del aire y del agua, y epidemias como la del VIH y posibles pandemias como la de gripe aviar o la gripe A por el virus H1N1, entre otros.

Ante la crisis actual, un nuevo modelo de desarrollo mundial se está incubando con nuevos actores y nuevas reglas de juego, y tiene que 
llevar a transformaciones en la salud pública, con una nueva lógica de análisis para encarar los problemas de la salud mundial y en enfrentar los determinantes sociales y políticos de la salud 23. Nuevos determinantes de la salud global se vienen reconociendo por fuerza de los cambios mundiales 24 . Es en este escenario, signado por múltiples desafíos y el cambio social, en el que la salud global se constituye en nueva disciplina para la interpretación y la acción en torno a la salud poblacional.

\section{Método}

Se trata de una revisión narrativa, siguiendo el método de análisis documental. Con el fin de establecer el estado del arte de perspectivas y tendencias de salud global, se construye una base de datos con información sobre los principales y más importantes centros, institutos y escuelas de salud global en el mundo y sus características.

Para la búsqueda se tuvieron en cuenta descriptores de salud global. La búsqueda se hace en PubMed, en LILACS y en Google Académico con las palabras clave: "salud global" y "global health". Posteriormente, se identifica autores de salud global y diplomacia global y la afiliación institucional correspondiente. Se complementa con los términos "centros de salud global", "global health centers", "saúde global”, "institutos de salud global", "global health institutes", "programas de salud global", "escuelas de salud global" y "global health programs".

Con la información obtenida, se crea una matriz que integra los diferentes centros, institutos y escuelas, detallando en ella nombre, dirección URL, dependencia; y se clasifican por tipo, carácter (académico/profesional, gubernamental, filantrópico), misión, perspectivas de salud global, país/región, que luego se resumen el la Tabla 1.

\section{Resultados}

Perfiles de las organizaciones académicas de salud global

Varios enfoques académicos se han perfilado acerca de la salud global, por parte de centros de formación e investigación, según diferentes países. A su vez, emergen varios intereses y orientaciones ideológicas, detrás de las concepciones de salud global.

En la pesquisa documental realizada para este trabajo, al identificar las tipologías de los centros de salud global se encuentra, en un abigarrado mosaico institucional, que unos son realmente centros, otros corresponden a institutos, escuelas, programas, alianzas, redes, fundaciones, entidades sin ánimo de lucro; organizaciones multilaterales, consorcios, negocios, coaliciones, proyectos, entre otros. La mayoría

Grupos y perspectivas de salud global en el mundo.

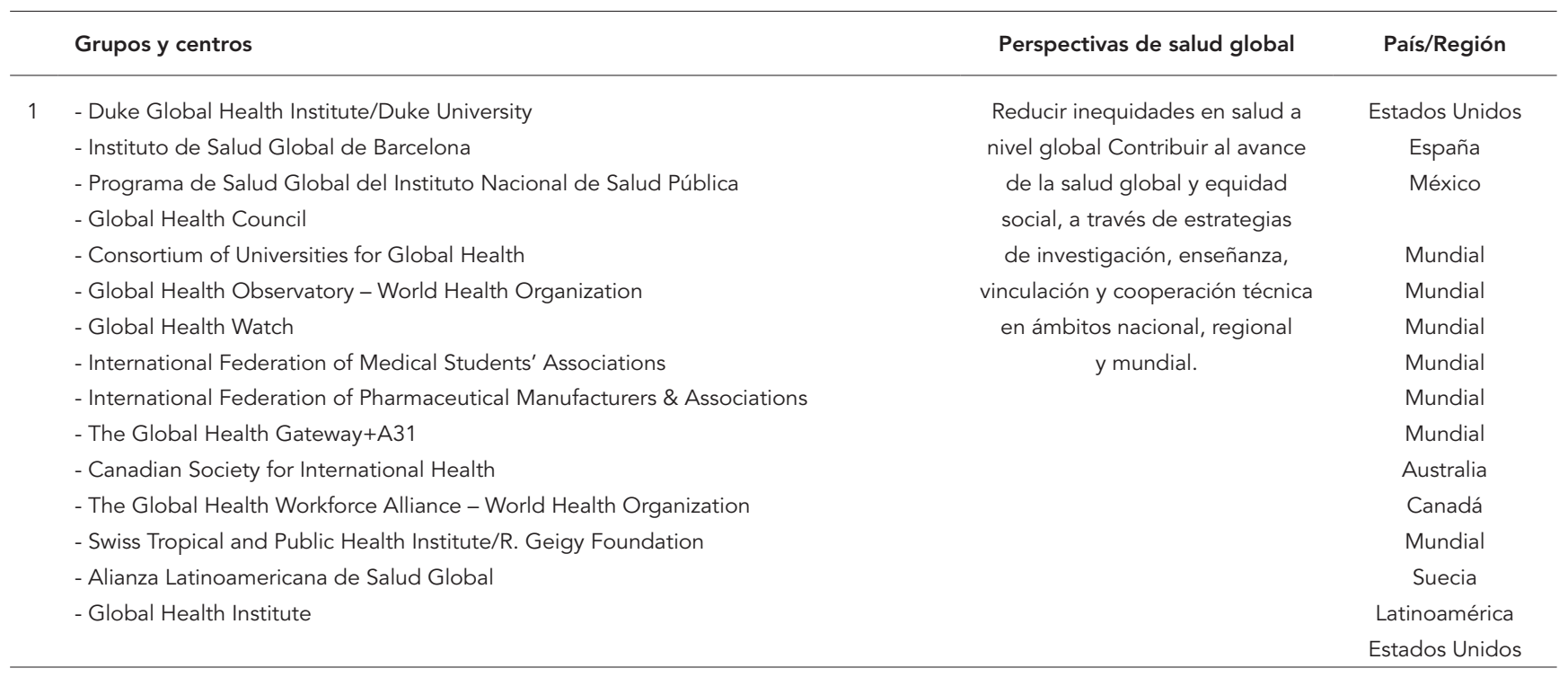

(continua) 


\begin{tabular}{|c|c|c|c|}
\hline & Grupos y centros & Perspectivas de salud global & País/Región \\
\hline \multirow[t]{19}{*}{2} & - The Canadian Coalition for Global Health Research & Responder a problemas de salud & Canadá \\
\hline & - Global Health Partnership Initiative & en poblaciones vulnerables & Estados Unidos \\
\hline & - Global Health Program - Bill and Melinda Gates Foundation & & Estados Unidos \\
\hline & - BIO Ventures for Global Health & & Estados Unidos \\
\hline & - Child and Family Health International & & Estados Unidos \\
\hline & - Global Health eLearning Center & & Estados Unidos \\
\hline & - U.S. Global Health Policy - Kaiser Family Foundation & & Estados Unidos \\
\hline & - JSI Research \& Training Institute, Inc. - Promoting and Improving Health/ & & Estados Unidos \\
\hline & John Snow Inc. & & \\
\hline & - Medical Care Development - Improving the Health and Well Being of People & & Estados Unidos \\
\hline & - Partners In Health & & Estados Unidos \\
\hline & - PATH - Program for Appropriate Technology in Health - A Catalyst for Global Health & & Estados Unidos \\
\hline & - The Rockefeller Foundation & & Estados Unidos \\
\hline & - Copenhagen School of Global Health/University of Copenhagen & & Dinamarca \\
\hline & - Johns Hopkins Center for Global Health/Johns Hopkins University & & Estados Unidos \\
\hline & - Karolinska Institute Centre of Global Health & & Suecia \\
\hline & - Network of institutions for Higher education in International Health & & Mundial \\
\hline & - The Federation of European Societies for Tropical Medicine and International Health & & Europa \\
\hline & - UCL Institute for Global Health/ University College London & & Inglaterra \\
\hline \multirow[t]{4}{*}{3} & $\begin{array}{l}\text { - Instituto de Investigación en Promoción y Educación para la Salud Global/Escuela de } \\
\text { Profesiones de la Salud, Recinto de Ciencias Médicas, Universidad de Puerto Rico }\end{array}$ & $\begin{array}{l}\text { Promover la salud global desde } \\
\text { perspectivas no tradicionales/por }\end{array}$ & Puerto Rico \\
\hline & - Action for Global Health & el mejor estado de salud posible. & Europa \\
\hline & - Doctors Without Borders & Trabajar por el bienestar & Mundial \\
\hline & - FHI360 - The Science of Improving Lives & de las personas & Estados Unidos \\
\hline \multirow[t]{7}{*}{4} & - Programa Salud Global Perú/Universidad Peruana Cayetano Heredia & Integrar la salud en las políticas e & Perú \\
\hline & - World Federation of Academic Institutions for Global Health & investigación a nivel transectorial & Estados Unidos \\
\hline & - The European Academic Global Health Alliance/The Association of Schools of Public & & Mundial \\
\hline & Health in the European Region & & Europa \\
\hline & - The International Association of Health Policy & & \\
\hline & - Global Health Programme/Graduate Institute of International and & & Mundial \\
\hline & Development Studies & & Suiza \\
\hline \multirow[t]{3}{*}{5} & - Global Health Delivery/Harvard University & Llevar servicios de salud y & Estados Unidos \\
\hline & - Global Health Research Center of Central Asia/Columbia University & promover reformas/ a países & Asia Central/ \\
\hline & & subdesarrollados & Estados Unidos \\
\hline \multirow[t]{4}{*}{6} & - Global Health - Centers for Disease Control and Prevention & Prevenir enfermedades y & Estados Unidos \\
\hline & - Global Health - United States Department of Health and Human Services & amenazas a la salud de & Estados Unidos \\
\hline & & los estadounidenses, & \\
\hline & & Seguridad Nacional & \\
\hline
\end{tabular}

de ellos ostentan características de académicos y profesionales, otros tienen algún componente gubernamental adicional como el Instituto de Salud Global de Barcelona, el programa de salud global de Perú, el Global Health Research Center of Central Asia, el Global Health- Center for Disease Control and Prevention, Global Health eLearning Center, Global Health ObservatoryWorld Health Organization, Medical Care Development- Improving the Health and Well Being of People, The Global Health Workforce Alliance-
World Health Organization; otros adicionalmente tienen un componente filantrópico, además del académico y/o gubernamental, pero siempre sobre una base de centro universitario académico o de investigación.

Para determinar los perfiles de salud global, se examinaron las misiones y propósitos de las instituciones encontradas, siendo sus fines más relevantes: reducir inequidades en salud a nivel global; promover la salud global desde perspectivas no tradicionales; responder a problemas de 
salud en poblaciones vulnerables; negociar por el mejor estado de salud posible; integrar la salud en las políticas e investigación a nivel transectorial; llevar servicios de salud y promover reformas en salud a países subdesarrollados; trabajar por el bienestar de las personas; prevenir enfermedades y amenazas a la salud de los estadounidenses; avanzar en los intereses de la Diplomacia Internacional; desarrollo y seguridad de los Estados Unidos. Todos, segú la misión de cada uno de los centros o instituciones.

De acuerdo con los propósitos de mayor alcance en los diversos centros estudiados (45), los podemos agrupar en 5 enfoques, con orientaciones muy diferentes: (a) "enfrentar problemas en las poblaciones vulnerables" (incluidos los servicios y reformas de los países en desarrollo) a lo cual se dirigen cerca de la mitad de los centros (20); (b) "reducir inequidades globales" (se inscriben la cuarta parte de los grupos investigados, en total (14); (c) en menor grado las misiones de los centros académicos se dirigen a "la investigación de las políticas transectoriales" (4); (d) "promover la salud global, el bienestar y mejorar el estado de salud de la población" (4); y (e) 2 más, propenden por la seguridad nacional de los Estados Unidos. Algunos centros contemplan más de uno de los propósitos clasificatorios.

De los centros estudiados, son de origen universitario y académico el $60 \%$, gubernamental, el $15 \%$ y no gubernamentales, el $25 \%$, aunque cerca de la mitad tienen la connotación mixta, bien sea gubernamental y académica, o agregando la alianza con algún organismo no gubernamental. Otra característica importante para analizar es la pertenencia a redes (o alianzas, consorcios $\mathrm{u}$ organismo multilateral en el que participa), siendo esta la tendencia mayoritaria (el 55\% de las consultadas); el resto (45\%) permanecen como centro de salud global con cierta autonomía (programas, proyectos, fundaciones, institutos o escuelas).

\section{Tendencias de los centros académicos de salud global}

A continuación, se describen algunas tendencias y actividades de algunos de los centros académicos seleccionados.

Así, el Programa de Salud Global de la Escuela de Salud Pública de la Universidad de Chile propende por una "salud pública sin fronteras" 5 . Se creó el Programa de Salud Global en el año 2010 , orientado a analizar los problemas clave en Salud Global en Latinoamérica, con el propósito de construir una agenda que apoye las políticas públicas en este campo 25 .
En Brasil, se ha tenido la iniciativa de formación en salud global y la diplomacia de la salud, en la Escuela Nacional de Salud Pública Sergio Arouca de la Fundación Oswaldo Cruz (ENSP/ Fiocruz) 26, ofrecido por pares externos e internos; no obstante, en la revisión que hacen los pioneros de esta iniciativa, encuentran debilidades conceptuales sobre los temas y señalan la carencia de una estructura de análisis sólida; otros más, señalan que los procesos de globalización son incontenibles y desatan nuevos desafíos para la cooperación y la salud internacional ${ }^{24}$. Al igual que la escuela de Fiocruz en Río de Janeiro, la Facultad de Salud Pública de la Universidad de São Paulo, también realiza actividades en salud global. El enfoque ha sido el de "salud global y diplomacia” y el impacto de la globalización sobre la salud y de la cooperación internacional en las políticas de salud de Brasil. También en São Paulo se ha considerado el tema de "bioética y salud global" 25 .

Por su parte, en el Perú, se desarrolla una iniciativa multidisciplinaria en la Universidad Peruana Cayetano Heredia (http://www.salud globalperu.org, accedido el 18/Feb/2015), encaminadaaladocenciaylainvestigaciónensaludglobal, enfatizando los problemas que enfrenta Perú en este campo y en el tema de diplomacia y salud global. Así como la cooperación internacional 25.

En México, en el Instituto Nacional de Salud Pública (INSP), se ha desarrollado un Grupo de Salud Global. De ellos retomamos uno de los conceptos que se discuten en este ensayo: la salud global es una manera de ver y abordar la salud, como un bien público mundial, como un tema de justicia social y como un derecho universal 27. Gira en torno a la equidad, la ética y los derechos humanos; a riesgos nuevos y re-emergentes; al trabajo con grupos poblacionales en desventaja social; a los retos comunes con profundidad local y alcance global; a la participación ciudadana; y a la interdisciplinariedad e intersectorialidad 28 . Desde el año 2003, el INSP ha impulsado la formación académica en temas de salud global (desafíos de la globalización y salud en América Latina, salud global en Latinoamérica). El programa se formaliza en el 200629.

Muchos de esos centros han avanzado de cursos de capacitación a diplomados, pasando a maestrías y doctorados en el área; es decir, que se va consolidando una masa crítica en salud global en la región.

La Facultad Nacional de Salud Pública de la Universidad de Antioquia en Colombia (Línea de investigación en salud global. http://www.udea. edu.co/portal/page/portal/SedesDependen cias/SaludPublica/E.Investigacion, accedido el 18/Feb/2015), ha constituido un grupo de salud 
global y una línea de investigación multidisciplinaria para el abordaje de los problemas globales, la salud global y las políticas públicas globales.

El debate académico em esta universidad ha llevado a precisar algunos aspectos que han contribuido a esta nueva área de salud global y a delimitar el tránsito de la salud internacional a la salud global 30 . Sus planteamientos son retomados en el desarrollo de este artículo y en los apartados siguientes. Más allá de la formación en seminarios y diplomados en salud global, así como la formación especializada en salud internacional, el énfasis se ha puesto en la investigación y en la teorización sobre salud global (una perspectiva conceptual y política).

Son muchas más las iniciativas latinoamericanas en este campo durante los últimos años. Como consecuencia de estas experiencias, los países Latinoamericanos y del Caribe, constituyen ALASAG (Alianza Latinoamericana de Salud Global). "ALASAG es una red de instituciones académicas que surge con el propósito de fortalecer el área de la salud global en la región y dar voz a los países de América Latina en espacios internacionales de discusión académica, de investigación y de políticas sobre el tema" 31 , se propone aportar la mirada latinoamericana a los temas más candentes de la agenda mundial sobre salud y desarrollo. Esta red entiende la salud global como "un bien público mundial que trasciende fronteras, (...) relacionado con la justicia social, y que tiene como ejes la equidad, la ética y el respeto a los derechos humanos" 31 . El fortalecimiento de esta alianza se ha logrado gracias a relaciones con otros pares internacionales tales como el Consorcio de Universidades para la Salud Global (CUGH), y ha participado en la creación de la Federación Mundial de Instituciones Académicas para la Salud Global en el 201325.

Por el lado europeo, actualmente en España, hay instituciones como la Escuela Andaluza de Salud Pública (Salud global. http://www. easp.es/category/salud_global/, accedido el 18/ Feb/2015) o la Escuela Nacional de Sanidad (Formación. http://www.isciii.es/ISCIII/es/conteni dos/fd-formacion/escuela-nacional.sanidad2. shtmil, accedido el 18/Feb/2015) que tienen áreas de salud global, y en Barcelona se encuentra el Instituto de Salud Global 32. Sin embargo, aún vemos que los programas transversales de salud Global, que tanta trascendencia y crecimiento han tenido en países como Estados Unidos, están ausentes en las universidades españolas 33 . No obstante, el grupo de Barcelona se plantea retos importantes en la salud global: la relaciona con los Objetivos de Desarrollo del Milenio y los nuevos Objetivos de Desarrollo Sostenible, así como la agenda post-2015 y señala 34: "que el nuevo paradigma de la salud global incluye un enfoque transdisciplinar para afrontar los problemas más acuciantes. (...) y trabajar juntos para obtener soluciones equitativas y sostenibles para los retos de la salud de hoy en día".

En otras regiones se han extendido varios grupos de salud global, dedicados a la investigación y a la asistencia social. Es el caso del grupo de Council on Health Research for Development (COHRED) 35 y el Foro Global de Investigación en Salud ${ }^{36}$, quienes utilizan una cartera de servicios y herramientas para apoyar a los países en el fortalecimiento de la gobernabilidad y la gestión de la investigación y la innovación para el desarrollo de la salud y la equidad socio-económica; también sirven en el área de la investigación y la innovación para la salud, así como el seguimiento de los flujos de recursos en la investigación en salud. Ayudan a los países de bajos y medianos ingresos en la construcción de la investigación y la innovación sostenibles dentro de los sistemas de salud.

Otros, como el Global Health Institute (GHI) de la Universidad de WisconsinMadison (Mission \& vision. http://ghi.wisc. edu/about/\#Mission\&Vision, accedido el 18/ Feb/2015), considera "la salud de los individuos y de las poblaciones a través de un marco integral de lugares saludables en los que la salud pública depende de los barrios y las políticas nacionales, con el estado del medio ambiente mundial". Este enfoque requiere la colaboración de todo el planeta completo para hacer frente a la atención sanitaria, la seguridad alimentaria y la agricultura sostenible, el agua y el saneamiento, la sostenibilidad ambiental y otras perspectivas por "una salud" que integra la salud de los seres humanos, los animales y el medio ambiente. El GHI aborda de raíz los determinantes sociales de la salud las causas de la mala salud en todos los sectores, buscando descubrir las estrategias más sostenibles para el bienestar humano.

Así tenemos muchos más centros, con perspectivas, misiónes y enfoques de la salud global muy diversos. Otros centros de salud global, como los ejemplificados, son sistematizados y se establecen sus perfiles, dejando ver su desarrollo colectivo e institucional, como se detallan al clasificarlos en cuatro (4) grupos en la Tabla 1.

\section{Discusión}

Este variopinto mosaico de centros consultados es sólo una muestra (Tabla 1) de lo que puede estar sucediendo en otros tantos a nivel mundial. Algunos de ellos, con sus enfoques dirigidos a enfrentar problemas en las poblaciones vulnerables (incluidos los servicios y las reformas de 
los sistemas de salud en los países en desarrollo), no distan en mucho de lo que ya históricamente en la segunda mitad del siglo XX se ha planteado en la disciplina de la salud pública, y se parecen mucho a modelos ya probados de salud internacional: modelos colonialistas, encaminados a intervenir en poblaciones del mundo subdesarrollado, muy propio del modelo clásico, que tenía como objeto de estudio e intervención los países en desarrollo y las minorías marginadas que viven en los países seguiendo, un enfoque "médico-biologista".

Otros centros se han planteado la renovación de la salud pública y la salud internacional, incluyendo algunos de los temas de actualización o el tema de la reforma sectorial, en lo cual se centró gran parte del debate de la salud pública y de los organismos multilaterales en la década de los 90.

Otros más, coinciden con quienes perciben los problemas de salud como responsabilidad de todos los países, sin importar su nivel de desarrollo, considerando las políticas internacionales como asunto de todos; propenden por "reducir inequidades globales", la investigación de las políticas transectoriales, y promover la salud global, el bienestar y mejorar el estado de salud de la población.

Finalmente estarían quienes han defendido una concepción crítica de la salud pública, modelos críticos, basados en las diferencias sociopolíticas entre los países industrializados y en desarrollo, concepción que sustentamos en el concepto de justicia global; haciendo eco también de las posturas basadas en la "reducción de la inequidades", el análisis de las "políticas transectoriales" y la necesidad de construir un concepto autónomo de salud global que nos guíe la acción política. Esta última perspectiva pretende englobar y asumir una visión latinoamericanista (como sería el caso de ALASAG).

Otros estudios y análisis también han hecho clasificaciones de los grupos y enfoques de la salud global, dando cuenta de una variedad de conceptos e interpretaciones también documentadas sobre la salud global en diferentes instancias y regiones. Así, Velji \& Bryant 10 presentan un recorrido conceptual de esta transdisciplina desde la salud individual hasta llegar a la salud global. Luego de reconocer un cambio en los valores, al decir que la Salud para Todos (la ambiciosa política de finales del siglo XX), no era simplemente una extensión de los valores anteriores, ni de las mismas estructuras y funciones; que las raíces de la salud global se daban en las tendencias sociales, propiciadas por técnicos y políticos que habían afectado el cuidado de la salud, tanto en los países menos desarrollados, como en los más desarrollados.
En consonancia, los autores describen la evolución de la salud global, en cuatro períodos de tiempo 10 en los cuales se produjeron esos cambios: la era de la salud individual, la era de la salud comunitaria, la era la salud de la población y la era de la salud mundial; pasando de concepciones basadas en la salud individual en hospitales y centros de salud con médicos y enfermeras, con enfoque de educación clínica, hasta llegar al estadio actual de la salud global, luego de pasar por la salud comunitaria y la salud poblacional. Hoy se impondría la comprensión mundial de la salud: se incluyen allí los Objetivos de Desarrollo del Milenio, los derechos humanos, la ética y el papel de los gobiernos; cubriendo no sólo a individuos, sino a comunidades y poblaciones a escala global; el tipo de instituciones también será diferente, mencionando los Centros Globales de Excelencia y los sistemas de salud construidos con equidad y calidad, atendidos esta vez por equipos integrados de salud. La formación y los valores tienen que ser con perspectivas mundiales, así como la enfermedad, no obstante, en el orden global es importante para los autores también la equidad y la cohesión social.

Por su parte, Solimano \& Valdivia concluyen en su pesquisa 25 (p. 361) que “...el discurso sobre Salud Global varía del tradicional altruismo de aliviar los problemas de salud de la población en los países pobres (similar al discurso de la salud internacional de principios y mediados del siglo $X X)$, a un discurso más auto referente de proyectarse como instituciones de excelencia en investigación y docencia en salud en el mundo".

En nuestro estudio, llama la atención que gran parte de los grupos angloparlantes (de los países del norte) han vuelto al tema de las enfermedades infecciosas, en su alcance global, y esto se centra en gran parte de lo que definen en la práctica por salud global. Otros también, desde la misma órbita geopolítica, quieren ir más adelante y proponer otras áreas de estudio. Así lo propone Velji 37 (p. 485) en su editorial: “Hay que encontrar soluciones al abordar otras prioridades de salud identificadas por los Objetivos de Desarrollo del Milenio. Una serie de organizaciones e iniciativas se han creado para satisfacer esta necesidad". Se propone desde esta óptica, el desarrollo de sistemas sólidos de salud, fortalecimiento de capacidades y educación, integrados, eficientes y equitativos, incorporando modelos de atención primaria y de mayor complejidad con enfoque de la investigación.

Sin duda, los nuevos temas han concitado nuevas perspectivas disciplinarias, pero habría que agregar, siendo más determinantes aún, las demandas del mundo globalizado, basado en la interacción y disminución de las distancias 
geográfico temporales que obliga a conocer el mundo y sus problemas más allá de las fronteras de sus países, y a establecer alianzas con sus pares y amigos diversos en todas las latitudes. Como ejemplo visible, se menciona la alianza de Universidades para la Salud Global (CUGH) y el Consorcio de Educación para la Salud Global (GHEC) 25, con más cien universidades de Estados Unidos y Canadá; también ocurre en el ámbito europeo y en América Latina con ALASAG.

Las razones ideológicas y políticas son determinantes y de hecho vienen perfilando esas tendencias de las que hemos hablado antes, bajo el liderazgo de algunos autores. Se resumen aqui: (1) la salud pública global como un bien público, basado en la diplomacia internacional, si seguimos a Kicbusch 38 y Kicbusch \& Kökény 39; (2) por otro lado, autores (Europa y países de este origen) que siguen una concepción más neutral frente a la salud pública convencional, así Koplan et al. 14 pueden estar en esta posición; (3) podría identificarse un enfoque norteamericano, asistencialista de la salud global, siguiendo a Farmer 40 y en parte a Frenk 41 (concepciones con arraigo en universidades como las de la Universidad de Harvard, la Universidad John Hopkins que pueden estar siguiendo esta línea), centrados en los sistemas de salud o preocupados, además, por un tema emergente, la seguridad nacional y mundial; (4) por último la versión latinoamericana que defendemos aquí, la cual busca su identidad en el ámbito de lo global, pero sin llegar a ser una confrontación con el norte, ni siquiera basada en la mera cooperación Sur-Sur, sino mucho más cosmopolita y anclada en valores universales, como se ampliará más adelante.

\section{Una perspectiva analítica}

Tan abigarrada exposición de experiencias, conceptos, tendencias y posiciones sobre salud global, nos enseñan cómo se van sentando las bases de configuración de la salud global como disciplina académica y objeto de estudio y análisis. Sin embargo, las concepciones son múltiples y tan diversas como grupos y escuelas se van configurando. Por ello, en lo que sigue de este análisis fijamos una visión que puede llegar a centrar la discusión, aunque no sea coincidente con muchas de las tendencias explicitadas.

El contexto es importante: la salud global está relacionada con el poder, los determinantes y el bienestar. Los temas en salud global deben referirse (como señalara Breilh 42 , en otro contexto de la salud pública) a una teoría de la necesidad, a los derechos humanos relacionados, a las diversas formas de interpretar la calidad de vida y sus determinantes, a los preceptos y los me- canismos de la seguridad humana, a cuestiones étnicas, raciales, de género y sociales de inequidad y poder. Estos temas han ganado espacio en las discusiones más recientes en Latinoamérica y en Europa y deben constituir ejes de análisis en el estudio de las políticas públicas y la salud en el ámbito global, acompañándose de los marcos epistémicos correspondientes y de los métodos de investigación e intervención política y práctica. Será importante entender esa transformación en salud, en función de lo global y de la dinámica sociopolítica de los diferentes actores en el espacio local, en defensa de sus derechos, de la salud y de la calidad de vida poblacional. Los determinantes sociales, nos han llevado a hablar de la "salud en todas las políticas" 43. No son las políticas de salud con enfoque sectorial las que garantizan el logro del bienestar y la salud, sino la acción mancomunada de la sociedad, mediante las políticas trans-sectoriales. Se trata de que las políticas de los diversos sectores tengan efectos favorables para la salud y no en contravía 44 .

Desde nuestro ángulo proponemos sistematizar las bases teóricas de la salud global en tres ejes, que sirvan para concertar algunas ideas, al respeto y para promover nuevas interpretaciones sobre la misma (aún desde perspectivas ideológicas diferentes).

(a) En primer lugar, el eje analítico de justicia, equidad y salud global: los avances teóricos en relación con la justicia global y la equidad, desde esta perspectiva se convierten en núcleo de desarrollo conceptual de la salud global para quienes la entendemos desde una postura democrática; pero a su vez es motivación política para las transformaciones necesarias de la salud mundial;

(b) En segundo lugar, el eje analítico de la acción transnacional y las políticas transectoriales por los derechos: se refiere a la gobernanza global y a la presencia de gobiernos eficaces para la salud, en su núcleo tenemos los derechos humanos y los derechos sociales en la época de la globalización, y cómo pasar de unos derechos atados al Estado Nación, para hacer la defensa de ellos en el nivel global. Se defienden los derechos humanos como una oportunidad para formulación de políticas públicas relacionadas con la salud 45 . (c) En tercer lugar, el holismo y la fuerza de una nueva conciencia global la visión holística y sistémica del mundo que abre nuevas posibilidades y nuevos desafíos para su interpretación, pero demanda de nosotros la innovación en nuevos desarrollos metodológicos e investigativos en la respuesta social de la salud pública global. Asimismo, recoge la idea de un sistema de salud mundial, en sintonía con el fortalecimiento de los sistemas nacionales de salud. 
Por limitaciones de espacio, no se ahonda en estos ejes de desarrollo de la salud global, pero pueden consultarse en otras fuentes de nuestro grupo $46,47,48$.

La salud pública global, desde su visión holística y sistémica nos reta a delinear algunos trazadores de política y nuevas intervenciones, basadas en una nueva cosmovisión y mega-cognición del mundo, necesarias tanto para la intervención en salud pública, como para renovar la investigación en esta área. En algunos estudios hemos dado cuenta de ese efecto global sobre la salud de las poblaciones 49,50,51. Pero no basta esta concepción técnica, sistémica y global de la salud, es necesario cambiar las prácticas y las intervenciones acordes con las nuevas interpretaciones y los nuevos enfoques metodológicos. Adicionalmente, es preciso entender desde la perspectiva de la determinación social y global, los problemas sociales y de la salud en el ámbito orbital o planetario actual.

En la Tercera Asamblea Mundial por la Salud de los pueblos en la Universidad de Western Cape, Ciudad del Cabo (Sudáfrica, julio de 2012) se preconizaba la "la formación de una conciencia global, [dado que] los modos de producir, de comerciar, de consumir, de aprender, de relacionarnos con la naturaleza, de comunicarnos y de hacer cultura, que el capitalismo ha producido, son lo opuesto de lo que debemos hacer por forjar el bienestar de la humanidad y proteger la vida sobre la tierra" 52 (p. 49).

Compete a la salud global esa transformación de la conciencia política, social y sanitaria y, en consonancia, las movilizaciones sociales por la salud y el bienestar en todo el mundo formarían parte de su arsenal, contribuyendo al desmonte de ese poder imperial, en lo cual están esperanzados varios de los pueblos del mundo.

La lucha por la equidad, la accesibilidad en salud y la defensa del derecho a la salud es importante, pero sus logros serán insuficientes, sino se dan en este contexto global. Contexto en el cual, varios problemas mundiales deben enfrentarse como desafíos ineludibles: el deterioro ambiental, dadas las prácticas industriales y financeiras destructivas (relación con el cambio climático). En el campo del sistema de salud, el complejo empresarial médico industrial en compañía de los procesos de reforma. Otro desafío en este contexto global es pasar de un derecho a la salud, basado en el acceso al servicio (por lo demás medicalizado), a una concepción integral de la salud que corresponda según Breilh 52 (p. 51): "a la lógica de los pueblos y del bien común y que asoma como parte sustancial del derecho a la salud, el disfrute de un nuevo modelo de civilización", lo cual se alcanzaría articulando "las luchas locales en el marco de una cohesionada movilización internacional" contra el tipo de civilización actual.

Un desafío no menos importante en el terreno de lo global (pasando por lo nacional y supranacional), y sin que estemos tratando de cubrir ni siquiera los más notables desafíos, es enfocar con criterio de renovación el actual sistema de gobernanza global, pero en particular el de la salud (parte de él son la Organización Mundial de la Salud y las agencias de cooperación internacionales), más aún si hacemos eco de los múltiples centros e instituciones estatales y no gubernamentales que hoy vienen actuando en la salud global, según este estudio. Conexo al anterior desafío se insta a la rendición de cuentas de los Fondos Públicos de la Cooperación Internacional, así como de sus agendas (muchas veces subordinadas a los intereses empresariales de los financiadores).

El anterior planteamiento, expuesto en "una perspectiva analítica, entra en sintonía con el enfoque "crítico del cosmopolitismo", el tercer enfoque que propusiera Palacio 53 (p. 7) para la justicia global, el cual se basa en las transformaciones sociales de la globalización, "en las experiencias de las reivindicaciones de justicia de colectivos y lo pueblos que denuncian los marcos regulativos fruto de la globalización económica". En tal sentido, se analizan las nuevas formas de sujeción, la transformación de la soberanía del Estado, la emergencia de nuevos actores internacionales (una nueva gobernabilidad) una nueva gobernanza. Enfoque que puede apoyarse en la versión de la teoría crítica de la Escuela de Frankfurt o en el análisis marxista del capitalismo, o en otra corriente filosófico-política, si pretendemos clarificar las luchas sociales de nuestro tiempo. Esta perspectiva cosmopolita es la más cercana al ideario de la salud global que preconizamos.

\section{Conclusión}

Varios enfoques académicos se han perfilado acerca de la salud global, por parte de centros de formación e investigación según diferentes países, como se vio en la primera parte de este artículo, los cuales resultan alentadores para continuar desarrollando esta veta de la salud pública mundial. Aunque se reconocen varios intereses y orientaciones ideológicas, detrás de la salud global, modificando sus concepciones según grupos privados, países desarrollados e instituciones académicas de países en desarrollo; también es cierto que el nuevo concepto se acompaña de varias propuestas de renovación en la salud mundial, tanto en el dominio episté- 
mico, como en el político social, todos van tras la construcción de un paradigma de renovación en la salud internacional y en la salud mundial, que dada su etapa preparatoria y las discusiones suscitadas, en lo que podemos llamar una etapa pre-paradigmática, no encuentra todavía su correlato final.

El enfoque de la salud global abre una oportunidad de cambio para la renovación de las es- trategias y políticas públicas actuales, la redefinición conceptual de la salud pública internacional, el avance de la globalización de la salud y la mitigación de los efectos de la globalización económica sobre los derechos humanos, elementos necesarios para el logro de una justicia global, razón última y componente esencial de la salud pública global.

\section{Resumo}

Visando estabelecer perspectivas da saúde global, este ensaio analisa diferentes tendências que enriqueceram, do ponto de vista acadêmico, a saúde global e internacional. Foi construída uma base de dados com informações sobre os principais e mais importantes centros de saúde global no mundo. Para a pesquisa, foram considerados autores de diplomacia global e de saúde global. A busca foi feita através de PubMed, LILACS e Google Scholar, com as seguintes palavraschaves: "salud global" $e$ "global health". Vários enfoques acadêmicos surgiram a respeito da saúde global, por parte de centros de ensino e pesquisa, em diversos países. Por sua vez, destacam-se vários interesses e orientações ideológicas, por trás do conceito de saúde global. O mosaico multifacetado dos centros de saúde global e de suas propostas sugere que esse novo conceito vai além da construção de um paradigma de renovação na saúde internacional e na saúde mundial, e que, em fase pré-paradigmática, ele ainda não encontrou uma versão final.

Saúde Global; Justiça Social; Políticas Públicas

\section{Agradecimientos}

Agradecimientos al comité de investigaciones de la Universidad de Antioquia (CODI) y a la estrategia de sostenibilidad 2013-2014, por el apoyo financiero y logístico. Al grupo de investigación en gestión y políticas públicas, línea de salud global, de la Facultad Nacional de Salud Pública (FNSP). A Daniel Tobón García, médico, Universidad Tecnológica de Pereira, pasante de internado rotatorio en la FNSP, por su contribución en la búsqueda y compilación de los centros e institutos de salud global. 


\section{Referencias}

1. Organización Mundial de la Salud. Revisión del reglamento sanitario internacional. Ginebra: Organización Mundial de la Salud; 2005.

2. Organización Mundial de la Salud. Informe sobre la salud en el mundo 2007. Panorama general. Un porvenir más seguro: protección de la salud pública mundial en el siglo XXI. Ginebra: Organización Mundial de la Salud; 2007.

3. Organización Panamericana de la Salud, Organización Mundial de la Salud. La salud y las relaciones internacionales: su vinculación con la gestión del desarrollo nacional de la salud. Washington DC: Organización Panamericana de la Salud/Organización Mundial de la Salud; 2008.

4. Douglas A. The international health partnership. Lancet 2007; 370:803-4.

5. Facultad de Medicina, Universidad de Chile. Programa de salud global. http://www.saludglobal. uchile.cl/home/index.php?option=com_content\& view $=$ category $\& i d=42 \& I t e m i d=57$ (accedido el 20/ Nov/2014).

6. Organización Internacional del Trabajo. Comisión mundial sobre dimensión social de la globalización. Por una globalización justa: crear oportunidades para todos. Ginebra: Organización Internacional del Trabajo; 2004.

7. Franco-Giraldo A, Álvarez-Dardet C. Salud pública global: un desafío a los límites de la salud internacional, a propósito de la epidemia de influenza humana A. Rev Panam Salud Pública 2009; 25:540-9.

8. Brown T, Cueto M, Fee E. The World Health Organization and the transition from "international" to "global" public health. Am J Public Health 2006; 96:62-72.

9. Organización Panamericana de la Salud. Competencias esenciales en salud pública: un marco regional para las Américas. Washington DC: Organización Panamericana de la Salud; 2013.

10. Velji A, Bryant JH. Global health: evolving meanings. Infect Dis Clin North Am 2011; 25:299-309.

11. Frenk J. 84th commencement address. http:// harvardmagazine.com/commencement/publichealth-commencementaddress (accedido el 24/ Nov/2014).

12. Institute of Medicine Committee on the U.S. Commitment to Global Health. The U.S. commitment to global health: recommendations for the public and private sectors. Washington DC: National Academies Press; 2009.

13. Brown C, Martineau F, Spry E and Yudkin JS. Postgraduate training in global health: ensuring UK doctors can contribute to health in resource-poor countries. Clin Med 2011; 11:456-60.

14. Koplan JP, Bond TC, Merson MH, Reddy KS, Rodríguez MH, Sewankambo NK, et al. Towards a common definition of global health. Lancet 2009; 373:1993-5.
15. Fried LP, Bentley ME, Buekens P, Burke DS, Frenk J, Klag MJ, et al. Global health is public health. Lancet 2010; 375:535-7.

16. Sanjuán H, Baquero H, Navarro E. Editorial. Salud UNINORTE 2011; 27:xi-i.

17. Kickbusch I, De Leeuw E. Global public health: revisiting healthy public policy at the global level. Health Promot Int 1999; 14:285-8.

18. Frenk J, Chacón F. Bases conceptuales para la educación e investigación en salud internacional. Washington DC: Organización Panamericanma de la Salud; 1992. (Human Resources Development Series, 95).

19. Patel V, Prince M. Global mental health: a new global health field comes of age. JAMA 2010; 303:1976-7.

20. Jacobsen KH. Introduction to global health. Burlington: Jones and Bartlett Publishers; 2008.

21. Velji A, Bryant JH. Global health ethics. In: Markle WH, Fisher MA, Smego RA, editors. Understanding global health. New York: McGraw Hill Medical; 2007. p. 295-317.

22. Burke DS. Foreword. In: Markle WH, Fisher MA, Smego RA, editors. Understanding global health. New York: McGraw Hill Medical; 2007. p. xv-i.

23. Marmot M, Friel S, Bell R, Houweling TA, Taylor S; Commission on Social Determinants of Health. Closing de gap in a generation: Health equity through action on the social determinants of health. Lancet 2008; 372:1661-9.

24. Franco-Giraldo A. Determinación global y salud: el marco amplio de los determinantes de la salud. Rev Fac Nac Salud Pública 2013; 31 Suppl 1:S73-86.

25. Solimano G, Valdivia L. Salud global en las instituciones académicas latinoamericanas: hacia un desarrollo e identidad propia. Saúde Soc 2014; 23:357-65.

26. Almeida CM. A experiência da Fiocruz na formação de profissionais em saúde global e diplomacia da saúde: base conceitual, estrutura curricular e primeiros resultados. RECIIS - Revista Eletrônica de Comunicação, Informação \& Inovação em Saúde 2010; 4:139-55.

27. Instituto Nacional de Salud Pública. Concepto de salud global. http://www.saludglobalinsp.mx/index.php?option $=$ com_content $\&$ view $=$ article $\&$ id $=10$ 6\&Itemid=367 (accedido el 04/Jun/2014).

28. Instituto Nacional de Salud Pública. Grupo de salud global. http://www.saludglobalinsp.mx/files/ Brochure_14x18\%20v_2_0_160712.pdf (accedido el 04/Jun/2014).

29. Salgado N. El programa de salud global del Instituto Nacional de Salud Pública. Cuernavaca: Instituto Nacional de Salud Pública; 2010.

30. Franco-Giraldo A. De la salud internacional a la salud global. Medellín: Editorial Universidad de Antioquia; 2011. 
31. Alianza Latinoamericana de Salud Global. ¿Qué es ALASAG? http://www.alasag.org/es/index.php/ preguntas-frecuentes/193-que-es-alasag (accedido el 18/Feb/2015).

32. Universitat de Barcelona. Nace el ISGlobal-Barcelona: el Instituto de Salud Global para luchar contra las enfermedades que afectan a las poblaciones más desfavorecidas. http://www.ub.edu/web/ub/ es/menu_eines/noticies/2010/02/48.html (accedido el 18/Feb/2015).

33. Bernardini D. Salud global en la era de las comunicaciones. Revista de Comunicación y Salud 2012; 2:35-6.

34. Instituto de Salud Global Barcelona. Punto de vista. http://www.isglobal.org/es/viewpoint-train (accedido el 18/Feb/2015).

35. Council on Health Research for Development. Supporting national health research systems in low and middle income countries. Geneva: Council on Health Research for Development; 2006.

36. Global forum for Health Research. Focusing research to improve global health. http://bibliodigi tal.saludpublica.uchile.cl:8080/dspace/handle/ 123456789/230 (accedido el 18/Feb/2015).

37. Velji A. Transforming global health, global health education, infectious disease, and chronic conditions in the 21st century. Infect Dis Clin North Am 2011; 25:485-98

38. Kickbusch I. Global health diplomacy: how foreign policy can influence health. BMJ 2011; 342:d3154.

39. Kickbusch I, Kökény M. Global health diplomacy: five years on. Bull World Health Organ 2013; 91:159.

40. Farmer P. Global health equity. Lancet 2004; 363 : 1832.

41. Frenk J. The Global Health System: strengthening national health systems as the next step for global progress. PLoS Med 2010; 7:e1000089.

42. Breilh J. Nuevos paradigmas en salud pública. In: Programa de Naciones Unidas para el Ambiente. Reunión internacional "Un enfoque de ecosistemas para la salud humana: enfermedades trasmisibles y emergentes". http://repositorio.uasb.edu. ec/bitstream/10644/3538/1/Breilh,\%20J-CON168-Nuevos\%20paradigmas.pdf (accedido el 18/ Feb/2015)
43. Etienne CF. Los determinantes sociales de la salud en las Américas. Rev Panam Salud Pública 2013; 34:377-8.

44. Guzmán MP. Deficiencias de los diagnósticos de las reformas sanitarias en los años 90 en América Latina. Rev Panam Salud Pública 2009; 25:84-92.

45. Franco-Giraldo A. Los derechos humanos, una oportunidad para las políticas públicas en salud. Gac Sanit 2008; 22:280-6.

46. Franco-Giraldo A. Equidad y salud: una perspectiva latinoamericana. In: III Congreso Latinoamericano de Salud Global. http://portal.fundevi. ucr.ac.cr/congresosaludglobal/recurses/e96f29_ III\%20CONGRESO\%20SALUD\%20GLOBALMEMORIA\%20DIGITAL.pdf (accedido el 18/Feb/ 2015).

47. López A, Franco-Giraldo A. Revisión de enfoques de política alimentaria: entre la seguridad y la soberanía alimentaria (2000-2013). Cad Saúde Pública 2015 ; 31:1355-69.

48. Franco-Giraldo A. Política pública, derechos sociales y globalidad. Medellín: Editorial Universidad de Antioquia; 2010.

49. Franco-Giraldo A, Álvarez-DArdet C, Ruiz MT. Effect of democracy on health: ecological study. BMJ 2004; 329:1421-3.

50. Franco-Giraldo A, Gil D, Álvarez-Dardet C. Tamaño del Estado (gasto público) y salud en el mundo, 1990-2000. Gac Sanit 2005; 19:186-92.

51. Franco-Giraldo A. Contexto político, Estado y salud en el mundo [Tesis de Doctorado]. Alicante: Universidad de Alicante; 2004

52. Breilh J. Más allá de la crisis actual: movilización por la salud para todos y todas. Medicina Social 2012; 7:49-54.

53. Palacio Avendaño M. Tres paradigmas de justicia global: estados, individuos y movimientos sociales. Coimbra: Centro de Estudos Sociais, Universidade de Coimbra; 2011. (Oficina do CES, 368).

Recibido el 22/Feb/2015

Aprobado el 10/Jun/2015 